\title{
On the heliolatitudinal variation of the galactic cosmic-ray intensity. Comparison with Ulysses measurements
}

\author{
G. Exarhos ${ }^{1,2}$ and X. Moussas ${ }^{1}$ \\ ${ }^{1}$ Laboratory of Astrophysics, Department of Astrophysics, Astronomy and Mechanics, School of Science, Faculty of Physics, \\ National and Kapodistrian University of Athens, Panepistimiopolis GR 15783, Zografos, Athens, Greece \\ ${ }^{2}$ Siemens Hellas A.E., Promitheos 12, Nea Kifisia, Athens, Greece
}

Received: 24 October 2002 - Revised: 21 April 2003 - Accepted: 20 May 2003

\begin{abstract}
We study the dependence of cosmic rays with heliolatitude using a simple method and compare the results with the actual data from Ulysses and IMP spacecraft. We reproduce the galactic cosmic-ray heliographic latitudinal intensity variations, applying a semi-empirical, 2-D diffusionconvection model for the cosmic-ray transport in the interplanetary space. This model is a modification of our previous 1-D model (Exarhos and Moussas, 2001) and includes not only the radial diffusion of the cosmic-ray particles but also the latitudinal diffusion. Dividing the interplanetary region into "spherical magnetic sectors" (a small heliolatitudinal extension of a spherical magnetized solar wind plasma shell) that travel into the interplanetary space at the solar wind velocity, we calculate the cosmic-ray intensity for different heliographic latitudes as a series of successive intensity drops that all these "spherical magnetic sectors" between the Sun and the heliospheric termination shock cause the unmodulated galactic cosmic-ray intensity. Our results are compared with the Ulysses cosmic-ray measurements obtained during the first pole-to-pole passage from mid-1994 to mid-1995.
\end{abstract}

Key words. Interplanetary physics (cosmic rays; interplanetray magnetic fields; solar wind plasma)

\section{Introduction}

Before the Ulysses' mission (launched October 1990) our knowledge about the heliolatitudinal variation of the cosmicray particles was limited to low heliographic latitudes arround the ecliptic plane. The cosmic-ray variations for various heliodistances was well studied from the measurements of the deep space probes Voyager 1, 2 and Pioneer 10, 11 (Venkatesan et al., 1984, 1985; Lockwood and Webber, 1981; Perko, 1995; Webber and Lockwood, 1995; Fujii and McDonald, 1997; McDonald et al., 2001; Potgieter and Ferreira, 2001).

Correspondence to: X. Moussas (xmoussas@cc.uoa.gr)
After the Jupiter flyby (February 1992) Ulysses continued its mission out of the ecliptic plane towards higher heliographic latitudes, measuring energetic particles and cosmic rays (Keppler et al., 1996; Quenby et al., 1996).

From mid-1994 to mid-1995 Ulysses completed its first pole-to-pole journey from $80^{\circ} \mathrm{S}$ to $80^{\circ} \mathrm{N}$ over the poles of the Sun, giving us for the first time the opportunity to study the latitudinal variation of many solar wind parameters (e.g. velocity, density, temperature, magnetic field) and the variation of cosmic-ray intensity. During this time period, close to solar minimum conditions, the temporal variations of the cosmic-ray intensity and the modulation level were relatively small. Since the Ulysses radial distance from the Sun varies between 1.3 AU and 2.3 AU during the pole-to-pole journey, the corrections due to radial cosmic-ray gradients are very small.

The Ulysses measurements revealed some unexpected features of the cosmic-ray intensity:

1. The latitudinal gradients were very small, $<1 \% / \mathrm{deg}$ for the galactic cosmic-rays (Simpson et al., 1996; Heber et al., 1998; Heber and Potgieter, 2000), although the background magnetic field tends to be nearly radial close to the poles, which means that the access of the cosmic-ray particles to the inner heliosphere would have been easier. It seems that transverse fluctuations in the high-latitude magnetic field (Forsyth et al., 1996) make the cosmic-ray particle access more difficult from the polar heliosphere (Jokipii and Kóta, 1989).

2. The symmetry plane of the cosmic-ray intensity appeared to be offset about $10^{\circ}$ from the heliographic equator to the south (Simpson et al., 1996).

3. The cosmic-ray intensity at the southern polar region is about $10 \%$ lower than the cosmic-ray intensity at the northern polar region. For more details, see McKibben et al. (1996), McKibben (1998), Fisk and Wenzel et al. (1998) and references therein. 


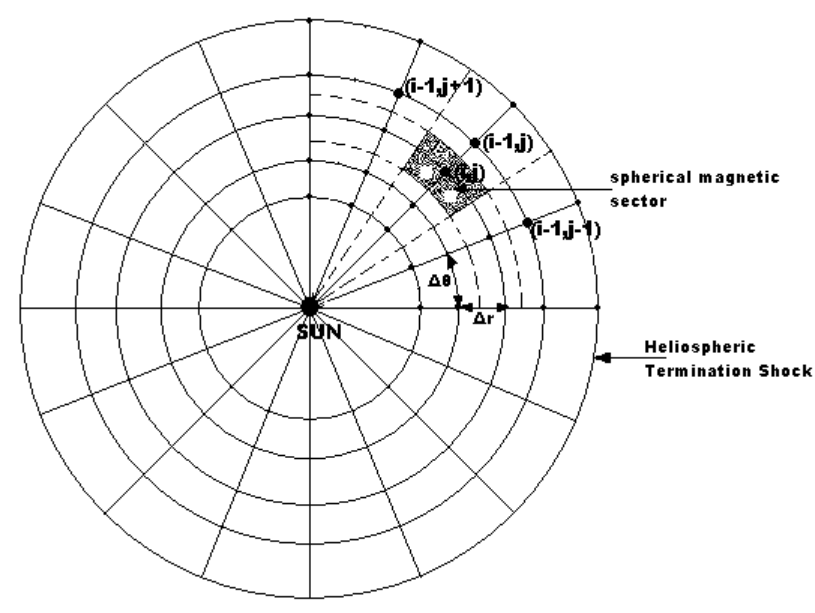

Fig. 1. Applied Heliospheric grid for the study of CR latitudinal variations. Definition of the "spherical magnetic sector".

In this work we reproduce the heliolatitudinal variation of the galactic cosmic-ray intensity, applying our shell-model (Exarhos and Moussas, 2001) and also including the latitudinal diffusion coefficient for the galactic cosmic-ray transport in the heliosphere. We use as input the Ulysses direct measurements of the heliospheric magnetic field. We assume Parkerian spatial variation of the heliospheric magnetic field. Finally, we compare our results with the observations.

\section{The 2-D shell-model}

We will briefly report on our shell-model for the cosmic-ray modulation. The basic idea of our model is that a magnetic region of certain dimensions modulates the cosmic-ray intensity exponentially as it travels to the outer heliosphere at the solar wind velocity

$J=J_{o} \exp \left(-\gamma u_{s w} B^{\alpha}\right)$

where $J_{o}$ and $J$ is the cosmic-ray intensity before and after the passage of the magnetic shell, $B$ is the mean magnetic field of the shell and $u_{s w}$ is the velocity of the magnetic shell that is frozen in the solar wind. The parameter $\alpha$ depends on the relationship of the diffusion coefficient $\kappa$ with the magnetic field $B$. Generally, this relationship is of the form $\kappa \propto 1 / B^{\alpha}$. A generally accepted value that we also use in our model is $\alpha=1$. The parameter $\gamma$ is chosen to give the best fit with the observations. However, the parameter $\gamma$ is not generally arbitrarily chosen, since its order of magnitude depends on the dimensions of the magnetic shell, that is $\gamma=D / \kappa_{0} B_{0}^{\alpha}$, where $D$ is the radial extension of the magnetic shell, $\kappa_{0} \sim 10^{12} \mathrm{~km}^{2-1}$ is the diffusion coefficient and $B_{0} \sim 1 \mathrm{nT}$ is the magnetic field at an arbitrary reference distance $r_{0}$ (i.e. $1 \mathrm{AU}$ ) (Exarhos and Moussas, 2001). The influence of the magnetic shell stops when it reaches the heliospheric boundary.

We extend now our previous 1-D model to 2-D, to include the latitudinal diffusion of the galactic cosmic rays. In this new model we divide the heliospheric region from the Sun up to the heliospheric termination shock into "spherical magnetic sectors", that are defined as solar wind plasma regions that have a small radial and latitudinal extension and are symmetric about the solar rotation axis. A spherical magnetic sector is a modulating region for the cosmic-ray intensity and it defines a grid point $(i, j)$ in our model (see Fig. 1).

We assume that the CR intensity $J(i, j)$ at the point $(i, j)$ is determined by the CR intensity of the three nearest outer grid points $J(i-1, j), J(i-1, j-1)$ and $J(i-1, j+1)$, as shown in Fig. 1. As in our 1-D model the influence of the spherical magnetic sector on the CR intensity as a traveling barrier stops when it reaches the heliospheric termination shock. Then, in a first order approximation, the cosmicray intensity at any point $(i, j)$ in the heliosphere can be expressed as

$$
\begin{aligned}
& J(i, j)=\left(J(i-1, j) \exp \left(-\gamma_{1} u_{s w} B_{(i-1, j)}^{\alpha}\right)\right. \\
& \quad+J(i-1, j-1) \exp \left(\gamma_{2} u_{s w} B_{(i-1, j-1)}^{\alpha}\right) \\
& \left.\quad+J(i-1, j+1) \exp \left(-\gamma_{3} u_{s w} B_{(i-1, j+1)}^{\alpha}\right)\right) / 3.0,
\end{aligned}
$$

where the index " $i$ " refers to the radial distance and the index " $j$ " refers to the heliospheric latitude. The index $i=1$ indicates the cosmic-ray intensity at the heliospheric boundary and increases as we travel from the heliospheric termination shock to the Sun, $i_{\max }=100$. The parameter $\gamma_{1}$ is the same as the parameter $\gamma$ we used in our 1-D model, that is $\gamma_{1}=\Delta r / \kappa_{0} B_{0}^{\alpha}$, where $\Delta r=D$ the radial extent of the magnetic sector or alternatively, the distance between the grid points $(i, j)$ and $(i-1, j), \kappa_{0}, \alpha$ and $B$ are given above. The parameters $\gamma_{2}$ and $\gamma_{3}$ in our model are assumed to be equal, and they are given by the expression $\gamma_{2,3}=f(i) \cdot \gamma_{1} . \delta$, where $f(i) . \Delta r=D_{i-1, j \pm 1}^{i, j}$ is the distance between the points $(i, j)$ and $(i-1, j \pm 1)\left(f(i)=\left[\left(i_{\max }-i\right)^{2}+\left(i_{\max }+1-i\right)^{2}-\right.\right.$ $\left.\left.2\left(i_{\max }-i\right)\left(i_{\max }+1-i\right) \cos (3.1415 . \Delta \theta / 180)\right]^{1 / 2}\right)$ and $\delta>1$. The parameters $\gamma_{1}$ and $\gamma_{2,3}$, so defined, are directly related to the diffusion coefficient $\kappa_{0}$ at the reference distance $r_{0}$. However, a direct relationship of the parameters $\gamma_{1}$ and $\gamma_{2,3}$ with the radial $\left(K_{r r}\right)$ and latitudinal $\left(K_{\theta \theta}\right)$ diffusion coefficient does not exist in our model.

We have to note at this point that Eq. (2) is not based on the definition of the radial $\left(G_{r}=\ln \frac{J\left(r_{1}, \theta\right)}{J\left(r_{2}, \theta\right)} /\left(r_{1}-r_{2}\right)\right)$ and latitudinal $\left(G_{\theta}=\ln \frac{J\left(r, \theta_{1}\right)}{J\left(r, \theta_{2}\right)} /\left(\theta_{1}-\theta_{2}\right)\right)$ gradients of the cosmic-ray intensity. From this definition we would have taken $\ln \frac{J(i, j)}{J(i-1, j-1)}=G_{r} \cdot \Delta r+G_{\theta} \cdot \Delta \theta$ (Webber and Lockwood, 1995), but this equation relates the CR intensity at the point $(i, j)$ only with the intensity at the point $(i-1, j-1)$. This is not the case for our model. We follow a different approach, and the parameters $\gamma_{1}$ and $\gamma_{2,3}$ are not equal to $G_{r}$ and $G_{\theta}$.

For the reproduction of the latitudinal variation of the galactic cosmic-ray intensity we apply a mesh of $100 \times 160$ grid points on the heliosphere from the Sun to the Heliospheric Termination Shock, assumed to be spherical with radius $R_{s}=100 \mathrm{AU}$ and consequently, we do not take into account the latitudinal variaton of the solar wind velocity. 


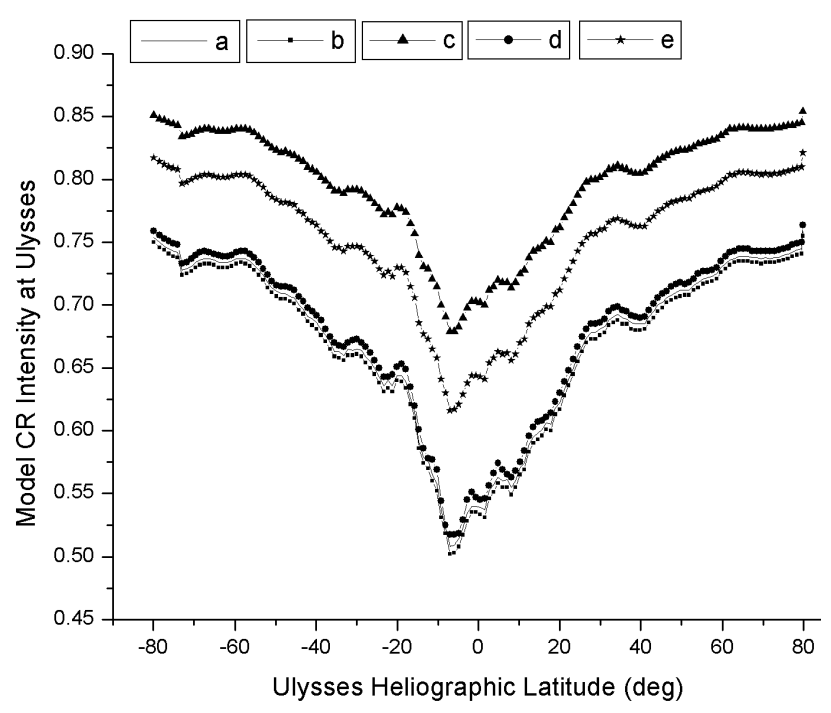

Fig. 2a. The heliolatitudinal variation of the cosmic-ray intensity for different values of the model parameters $\gamma_{1}$ and $\gamma_{2,3}$ a) $\gamma_{1}=$ $1.75 e^{-5}$ and $\left.\delta=10 b\right) \gamma_{1}=2.5 e^{-5}$ and $\delta=7$ c) $\gamma_{1}=1.0 e^{-5}$ and $\delta=10 d$ ) $\gamma_{1}=1.25 e^{-4}$ and $\delta=10$ and $e$ ) $\gamma_{1}=1.25 e^{-5}$ and $\delta=10$;

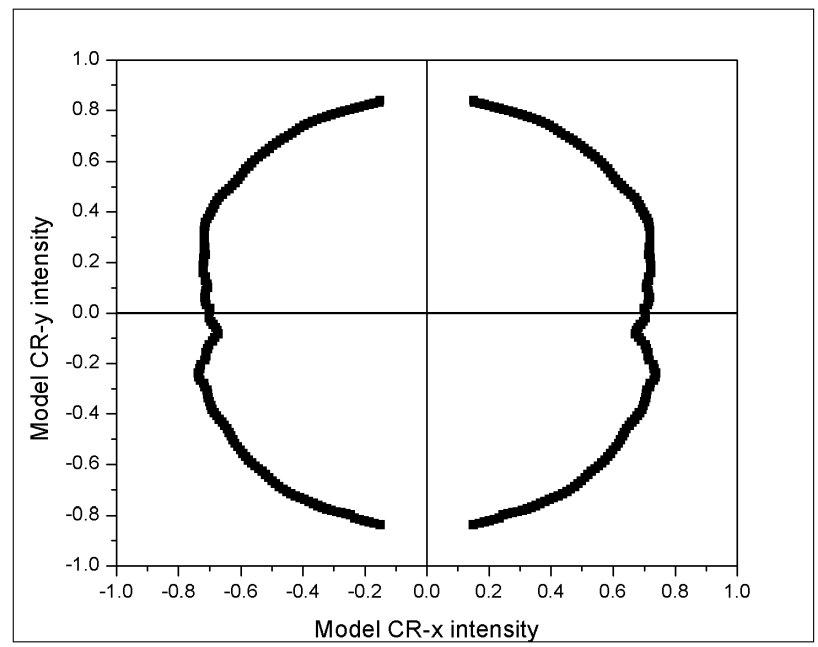

Fig. 2b. The heliolatitudinal profile of the cosmic-ray intensity obtained from the model for $\gamma_{1}=1.0 e^{-5}$ and $\delta=10$.

Our model has rotational symmetry about the solar axis. Each spherical magnetic sector has radial width $\Delta r=1 \mathrm{AU}$ and latitudinal width $\Delta \theta=1 \mathrm{deg}$ (the Ulysses heliolatitude varies from $-80 \mathrm{deg}$ to $+80 \mathrm{deg}$ ). The magnetic field we use is the 1 deg average value of the Ulysses direct measured magnetic field. The magnetic field at each grid point $(i, j)$ is the extrapolated average magnetic field using the Parkerian model.

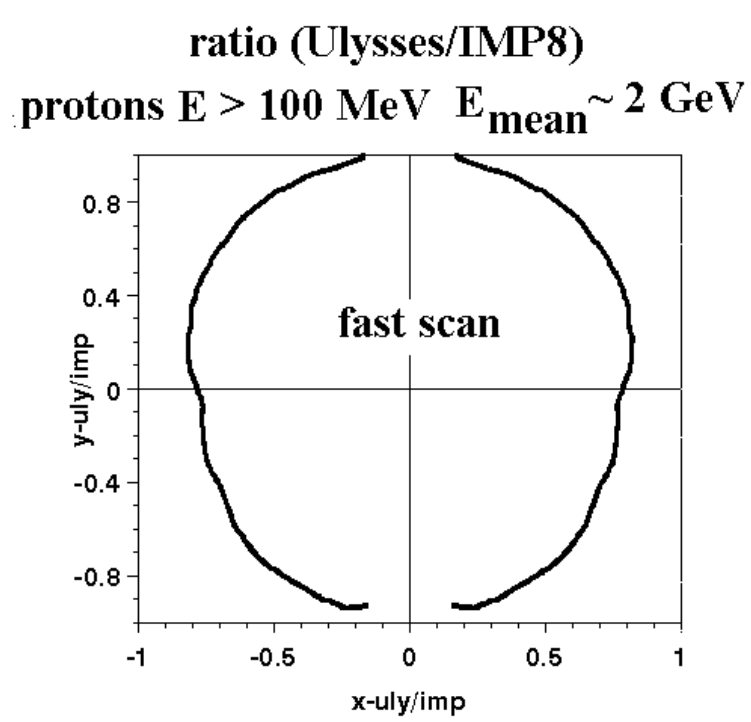

Fig. 3a. The Ullysses cosmic-ray heliolatitudinal variation;

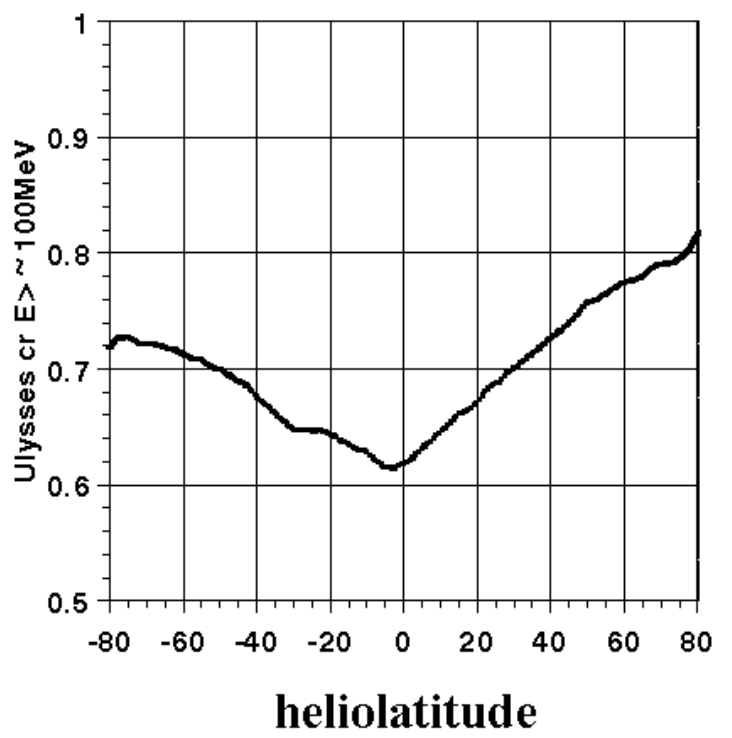

Fig. 3b. The Ullysses cosmic-ray intensity profile.

\section{The model results}

Using 1 deg averages from the hourly values of the heliospheric magnetic field from the Ulysses spacecraft during its first pole-to-pole passage, we reproduce the latitudinal variation of the integrated galactic cosmic-ray intensity shown in Fig. 2. For these results we assume that the radius of the heliospheric boundary is $R_{S}=100 \mathrm{AU}$. We do not include the latitudinal variations of the solar wind velocity, and we use a constant value $u_{s w}=400 \mathrm{~km} / \mathrm{s}$. The cosmic-ray intensity is normalized to the unmodulated intensity out of the heliospheric boundary. In Fig. 3 we show the Ulysses CR data $(E>100 \mathrm{MeV})$. Comparing Fig. 2 with Fig. 3 we see 
that our model can reproduce not only the $\sim 10^{\circ}$ offset of the modulation surface, to the south of the heliographic equator, as first reported by Simpson, Zhang and Bame (1996), but also the general heliolatitudinal profile of the cosmic-ray intensity.

Another interesting result is that our model gives an average latitudinal gradient $G_{\theta} \sim 0.36 \% /$ deg for $\gamma_{1}=1 e^{-5}$ and $\delta=10$, which is almost equal to the cosmic-ray latitudinal gradient reported by Simpson, Zhang and Bame (1996), obtained from the Ulysses/IMP-8 daily averages of energetic particles with energy $E \geq 100 \mathrm{MeV}$ (mean energy $E \sim 2 \mathrm{GeV}$ ), if we take the modulation symmetry surface to be at a heliographic latitude of $\sim 10^{\circ}$ to the south.

The average radial gradient $G_{r}$ obtained from our model for $\gamma_{1}=1 e^{-5}$ and $\delta=10$ is approximately equal to $\sim 1.3 \% / \mathrm{AU}$, while the radial gradient obtained from Ulysses is $\sim 2.5 \%$ /AU. During the in-ecliptic period of Ulysses' journey from 1 to $5.4 \mathrm{AU}$, Heber et al. (1993) estimated the radial gradial to be $\sim 0.3 \% / \mathrm{AU}$ for protons with energy $>106 \mathrm{MeV}$. Our model, for the ecliptic zone, gives a radial gradient $\sim 3 \% / \mathrm{AU}$, which is an order of magnitude greater from the one reported. However, due to the small variation of the Ulysses orbital radius during the fast latitude scan and the problems of re-normalization with IMP-8 data (see footnotes in McKibben, 1998), the errors of the estimated radial gradients obtained from the Ulysses measurements for high heliolatitudes are very large.

The latitudinal gradients of the cosmic-ray intensity obtained from our model for the different latitudinal intervals were calculated using a least-square fit of the cosmic-ray intensity values $J\left(i_{\max }, j\right)$ as a function of the heliolatitude, where $i_{\max }=100$ corresponds to $r=1 \mathrm{AU}$ and $j \in[1,160]$ runs for the different heliolatitudes.

\section{Conclusions}

We have shown that the heliolatitudinal profile of the galactic cosmic-ray intensity that has been observed by the Ulysses spacecraft can be reproduced if we apply a 2-D diffusionconvection model that includes the radial and latitudinal diffusion of the cosmic-ray particles.

The variation of the heliospheric magnetic field and its configuration seems to be the major modulating factor for the cosmic-ray intensity. By using as the only variable in our model the Ulysses measurements of the heliospheric magnetic field during the so-called fast-scan, we can reproduce not only the $\sim 10^{\circ} \mathrm{S}$ offset of the modulation symmetry surface, but also a latitudinal gradient of the cosmic-ray intensity very close to the observed one.

In this model the radius of the heliospheric boundary and the solar wind velocity do not vary with the heliolatitude. We have not included all the parameters that affect the cosmicray intensity (e.g. the rigidity of the particles, the gradient and curvature drifts, etc.), since the aim of our work is to see if a semi-empirical, 2-D diffusion-convection model can reproduce the observed cosmic-ray profile. Since the energy variations of the particles are not included in this model, we may take the calculated cosmic ray intensity as the integrated cosmic ray flux over particle energy (or rigidity). We believe that the method we have developed in this work provides a simple way to reproduce the heliolatitudinal profile of the galactic cosmic ray intensity, avoiding the much more complicated numerical models and simulations.

Acknowledgements. We express our gratitude to the University of Athens for providing the Grant 70/4/2469 for Space Physics. We thank the University of Chicago and especially R. B. McKibben for the high energy particles data of Ulysses spacecraft.

Topical Editor R. Forsyth thanks two referees for their help in evaluating this paper.

\section{References}

Exarhos, G., and X. Moussas: Simulation of the temporal variations of the galactic cosmic-ray intensity at neutron monitor energies, Solar Physics, 200, 283, 2001.

Fisk, L. A., Wenzel, K.-P., Balogh, A., Burger, R. A., Cummings, A. C., Evenson, P., Heber, B., Jokipii, J. R., Krainev, M. B., Kóta, J., Le Roux, J. A., McDonald, F. B., McKibben, R. B., Potgieter, M. S., Simpson, J. A., Steenberg, C. D., Suess, S., Webber, W. R., Wibberenz, G., Zhang, M., Ferrando, P., Fujii, Z., Lockwood, J. A., Moraal, H., and Stone, E. C.: Global Processes that Determine Cosmic Ray Modulation, Space Sci. Rev., 83, 179, 1998.

Forsyth, R. J., Balogh, A., Horbury, T. S., Erdös, G., Smith, E. J., and Burton, M. E.: The heliospheric magnetic field at solar minimum: ULYSSES observations from pole to pole, Astron. Astrophys., 316, $24201,1996$.

Fujii, Z. and McDonald, F. B.: Radial intensity gradients of galactic cosmic rays (1972-1995) in the heliosphere, J. Geophys. Res., 102, 24 201, 1997.

Heber, B., Raviart, A., Paizis, C., et al.: Modulation of galactic cosmic ray particles observed on board the ULYSSES Spacecraft, Proc. 23rd International Cosmic ray Conference (Calgary), 3, 461, 1993.

Heber, B., Bothmer, V., Droege, W., Kunow, H., Mueller-Mellin, R., Sierks, H., Wibberenz, G., Ferrando, P., Raviart, A., and Paizis, C.: Latitudinal distribution of greater than $106 \mathrm{MeV}$ protons and its relation to the ambient solar wind in the inner southern and northern heliosphere - ULYSSES Cosmic and Solar Particle Investigation Kiel Electron Telescope results, J. Geophys. Res., 103, 4809, 1998.

Heber, B. and Potgieter, M. S.: Galactic Cosmic Ray Observations at Different Heliospheric Latitudes, Adv. Space Res., 26, 839, 2000.

Jokipii, J. R. and Kóta, J.: The polar heliospheric magnetic field, Geophys. Res. Lett., 16, 1, 1989.

Keppler, E., Drolias, B., Fraenz, M., Korth, A., Reuss, M. K., Blake, B. and Quenby, J. J.: The high latitude pass of ULYSSES: energetic particle observations with EPAC, Astron. Astrophys., 316, 464, 1996.

Lockwood, J. A. and Webber, W. R.: A study of the long-term variation and radial gradient of cosmic rays out to $22 \mathrm{AU}, 17$ th ICRC, Paris, France, July 13-25, 1981, Conference paper, vol. 1, 259262, 1981. 
McDonald, F. B., Cummings, A. C., Lal, N., McGuire, R. E., and Stone, E. C.: Cosmic rays in the heliosphere over the solar minimum of cycle 22, Proceedings of ICRC 2001, 3830, 2001.

McKibben, R. B., Connel, J. J., Lopate, C., Simpson, J. A., and Zhang, M.: Observations of galactic cosmic rays and the anomalous helium during ULYSSES passage from the south to the north solar pole, Astron. Astrophys., 316, 547, 1996.

McKibben, R. B.: Three-dimensional Solar Modulation of Cosmic Ray and Anomalous Components in the Inner Heliosphere, Space Sci. Rev., 83, 21, 1998.

Perko, J. S.: Time-dependent modulation of galactic cosmic rays by merged interaction regions, J. Geophys. Res., 98, 19 027, 1995.

Potgieter, M. S. and Ferreira, S. E. S.: Modulation of cosmic rays in the heliosphere over 11 and 22 year cycles: a modelling perspective Adv. Space Res., 27, 481, 2001.

Quenby, J. J., Drolias, B., Keppler, E., Reuss, M. K., and Blake,
J. B.: A "Ulysses" Cosmic-Ray Latitude Gradient Observation and Modulation Models, Solar Phys., 163, 397, 1996.

Simpson, J. A., Zhang, M., and Bame, S.: A Solar Polar NorthSouth Asymmetry for Cosmic-Ray Propagation in the Heliosphere: The ULYSSES Pole-to-Pole Rapid Transit, Astrophys. J., 465, L69, 1996.

Venkatesan, D., Decker, R. B., and Krimigis, S. M.: Radial gradient of cosmic ray intensity from a comparative study of data from Voyager 1 and 2 and IMP 8, J. Geophys. Res., 89, 3735, 1984.

Venkatesan, D., Decker, R. B., Krimigis, S. M., and van Allen, J. A.: The galactic cosmic ray intensity minimum in the inner and outer heliosphere in solar cycle 21, J. Geophys. Res., 90, 2905, 1985.

Webber, W. R. and Lockwood, J. A.: Intensity variations of $>70$ Mev cosmic rays measured by Pioneer 10, Voyager 1 and 2, and IMP in the heliosphere during the recovery period from 19921995, Geophys. Res. Lett., 22, 2669, 1995. 